\title{
Ruler-based mechanisms of ribonuclease III enzymes
}

Xinhua Ji

National Cancer Institute, Frederick, Maryland, USA

Ribonuclease III (RNase III) represents a family of dsRNA-specific endoribonucleases required for RNA maturation and gene regulation. Bacterial RNase III and eukaryotic Rnt1p, Dcr1, Drosha, and Dicer are representative members of the family. Our laboratory has been studying the structure and mechanism of RNase III enzymes since 1996. We have reported a total of eleven crystal structures of bacterial RNase III in complex with dsRNA at various catalytic stages of the enzyme, including the first structure of a catalytically meaningful RNase III-RNA complex (Cell, 124:355-366, 2006) and the structure of a catalytic stage immediately after the cleavage of the phosphodiester bond (Mol Macrobiol, 67:143-154, 2008). We have also determined the crystal structure of a post-cleavage complex of Rnt1p from yeast, the first structure of a eukaryotic RNase III in complex with RNA in a catalytically meaningful manner (Molecular Cell, 54:431-444, 2014). Strikingly, the structure features two rulers for substrate selection. This doubleruler mechanism represents an example of the evolution of substrate selectivity and provides a framework for understanding the catalytic mechanism of eukaryotic RNase IIIs.

The worldwide effort in structural analysis of other eukaryotic RNase III enzymes resulted in several important structures, including the crystal structures of Giardia intestinalis Dicer (Science, 311, 195-198, 2006), Kluyveromyces polysporus Dcr1 (Cell, 146, 262-276, 2011), and human Drosha (Cell, 164, 1-10, 2016). These structures, however, do not contain RNA and thus are not able to explain their mechanisms of action. Our structures of bacterial RNase III greatly enhanced the significance of these eukaryotic RNase III structures. On the basis of protein-RNA interactions revealed by our structures, models with RNA can be reliably constructed for these RNase IIIs. A model complex of Dicer with RNA explains how Dicer enzymes recognize the 2-nt 3' overhang of the dsRNA substrate and measure $22 \mathrm{nt}$ up to position the scissile bond over the cleavage site. A model complex of Dcrl with RNA explains how homodimers of noncanonical Dicer enzymes bind cooperatively along dsRNA such that the distance between active centers in adjacent RIIID dimers is the length of $22 \mathrm{nt}$. A model complex of Drosha with RNA explains how Drosha enzymes recognize the last base pair in the basal junction of the primary microRNA substrate and measure $11 \mathrm{nt}$ up to position the scissile bond over the cleavage site. 